\title{
Performance Enhancement of MIMO System with Varying the Number of Transmitting and Receiving Antennas
}

\author{
VijayaDurga Ravva, Allan McLauchlin
}

\begin{abstract}
This paper current limit and execution of different reception apparatuses for remote correspondence frameworks. Numerous radio wires structures are classified into single-input various yields (SIMO), different sources of info single yield (MISO), and numerous data sources numerous yields (MIMO) frameworks. Expecting that the channel is obscure at beneficiary, articulations for the limit are given for each structure. The impact of utilize dissimilar reaction apparatuses on the limit of remote correspondence frameworks by utilizing Matlab code. Our outcomes demonstrate that expanding the quantity of transmitting and accepting radio wires for a remote MIMO channel improves the channel limit that can be acquired. Since mixed media application requires higher information rate which can be conceivable by MIMO (8x8) framework, in this paper Channel limit of the (8x8) MIMO is evaluated and contrasted and channel known and obscure. It has been demonstrated the reliance of MIMO channel limit on channel information. Future remote correspondence frameworks play out a leap forward in framework execution, by utilizing radio wire clusters at the two sides of correspondence connect.
\end{abstract}

Catchphrases: MIMO Structures; Channel Capacity; SNR; SISO; SIMO; MISO; MIMO Channels; SNR.

\section{INTRODUCTION}

Through the previous decade, Wireless Communications particularly portable correspondence frameworks have emerged drastically. The radio correspondence frameworks must meet the necessities of developing number clients no less interest for new administrations. New computerized innovations are currently being created decide fifth era versatile correspondence frameworks for guaranteeing high ghastly effectiveness and broad group of administrations. In this situation, correspondence frameworks utilizing reception apparatus demonstrate at transmitter and beneficiary are capable of providing rapid transmission with a base nature of administration guaranteed. The exceptional research on MIMO frameworks was motivated thorough works by Telatar [11] Foschini and Gans [12] that demonstrated sensational direct increment in channel limit with quantity reception apparatuses [13]. MIMO frameworks encourage us work two unmistakable elements radio connection; first being Diversity and second being Capacity.

Revised Manuscript Received on October 05, 2019.

Vijaya Durga Ravva, Department of Electronics and Communication Engineering, University of Hertfordshire, College Ln, Hatfield AL10 9AB, UK.

Allan McLauchlin, Department of Electronics and Communication Engineering, University of Hertfordshire, College Ln, Hatfield AL10 9AB, UK.
Inthis paper we will examine Capacity MIMO channels because it is exhibition measure for computerized correspondence frameworks it is maximal transmission rate for which solid, secure quick correspondence can be achieved. This paper is sorted out as pursues. Introduction SISO,MISO, SIMO MIMO Systems is appeared (Section II).

At that point we will examine Capacity for all frameworks we will focuson MIMO channels limit (Section III) and we will finish (Section IV) by an examination between various multi-radio wire structures addition on limit by expanding quantity reception apparatuses and contrasted channel limit channel known and obscure.

\section{MIMO SYSTEMS (SISO-MISO/SIMO-MIMO)}

There are a few organizations of MIMO which can be utilized from SISO, through SIMO and MISO to the full MIMO frameworks [6]. These can give critical upgrades of execution, yet by and large at rate of extra handling and the a greater amount of radio wires utilized. Parities of execution against costs, size, preparing accessible and the subsequent battery life should be made when picking the correct alternative. Various information and numerous yield, called as MIMO is exploitation diverse radio wires at both the transmitter and collector for the improvement of communication performance. MIMO frameworks can be viewed as a next form of keen receiving wires, an efficient mechanism utilizing reception apparatus exhibits for improving execution of remote transmission [1]. That likewise gives favorable circumstances over single-radio wire frameworks, both channel limit bit mistake rate. MIMO frameworks, that utilization different component radio wires for transmission and gathering sign, are relied upon to assume key job in improving exhibition future remote correspondence frameworks [2], [3]. It has been clear that if sign blurring between sets transmit get receiving wire components is free indistinguishably dispersed (i.i.d.), limit MIMO frameworks can increment directly with quantity of reception apparatuses [4]. Various information different yield, or MIMO [5], is radio interchanges innovation or RF innovation which is being referenced and utilized in numerous new advances now days. Wi-Fi, LTE; Long haul Evolution, various other radio, remote $\mathrm{RF}$ advances are using the new MIMO remote development to achieve extended association limit ghostly effectiveness joined with improved connection unwavering quality through loud ways.

\section{Published By:}


A MIMO framework contains $\mathrm{N}$ transmitting radio wires and $\mathrm{M}$ accepting recieving wires appeared in (1), $\mathrm{x}$ is vector with $\mathrm{N}$ images

$$
\mathrm{X}=\left[\mathrm{x}_{1} \ldots \mathrm{x}_{\mathrm{t}} \ldots \mathrm{x}_{\mathrm{N}}\right]^{\mathrm{T}}
$$

$\mathrm{H}$ is channel matrix of size $\mathrm{N} \times \mathrm{M}$. [6],

$$
\begin{aligned}
& \text { [7], [14] } \\
& \mathrm{H}=\left[\begin{array}{ccc}
\mathbf{h}_{11} & \cdots & \mathbf{h}_{1 \mathrm{~N}} \\
\vdots & \ddots & \vdots \\
\mathrm{H}_{\mathrm{M} 1} & \cdots \mathrm{H}_{\mathrm{mn}}
\end{array}\right]
\end{aligned}
$$

focuson the limit of MIMO channels Fig.1 and Fig.2. As we probably am aware Capacity,a execution measure for computerized correspondence frameworks is the maximal transmission rate for which a dependable correspondence can be accomplished [8]. On the off chance that the transmission rate gets bigger than thecapacity, the framework separates and the collector begins executing translating mistakes with a nonimmaterial likelihood. Capacityis the essential apparatus to describe the exhibition of MIMO frameworks and it additionally serves in useful framework as a kind of perspective to appropriately plan the transmitted sign and the handling of the got signals[8]. Multi-reception apparatus channel hnm corresponding to the channel between nth transmission radio wire and $\mathrm{mth}$ reception recieving wire. (Fig.1)

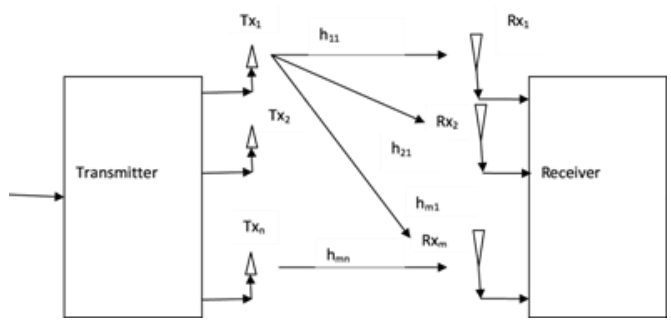

Fig.1. MIMO system $\mathrm{N} \times \mathrm{M}$

We consider commotion vector $\mathrm{b} \mathrm{M}$ accepting recieving wires suchthat:

$\mathrm{b}=\mathrm{b} 1 \ldots \mathrm{bt} \ldots \mathrm{bNT}(3)$

At that point got vector $\mathrm{y}$ is given as pursues:

$\mathrm{y}=\mathrm{Hx}+\mathrm{b}$

It is commonly expected that clamor is immaterial to have option to recreate transmitted sign. The articulation (4) can be composed:

$\mathrm{y}=\mathrm{Hx} \quad$ (5)

wheny and Hare known, the relationship which permits recreation $\mathrm{x}$ is:

$\mathrm{x}=\mathrm{H}-1 \mathrm{y} \quad(6)$

Numerically, fathoming this condition needs that $\mathrm{H}$ is invertible which implies that $\mathrm{H}$ is full position (its size is equivalent to its position). Physically, this outcomes in constriction and diverse stage movements endured by each transmitted sign. This is the reason it is important to have a situation rich in multipath to exploit the commitments of a MIMO framework.

\section{SYSTEM STRUCTURE OF SISO, MISO, SIMO ANDMIMO CHANNEL}

Similarly we will outline a correlation between the distinctive multi-reception apparatus frameworks; the motivation behind this area is to assess how the transmission capacitychanges as far as radio wire number. Here we will look at limit various MIMO channels (SISO, SIMO, MISO and MIMO) without knowing about territory CSI channel. Thought channel Capacity was presented by Claude Shannon by articulation (7) regularly known as Shannon's formula[9]-[11]:

$\mathrm{C}=\mathrm{W} \log 2(1+\mathrm{P} / \mathrm{N})$ bits/second.
A logarithmic capacity for what number bits data that can be transmitted without mistake every second over channel with transfer speed $\mathrm{W}(\mathrm{Hz})$ and indication to clamor quantity $(\mathrm{P} / \mathrm{N})$.

\section{A. Capacity of SISO Channel}

SISO system with channel gain h, signal to noise ratio SNR at receiving antenna, capacity without knowledge about the CSI: $\mathrm{C}=\log _{2}(1+$

$$
\operatorname{SNR}|\mathrm{h}|^{2} \text { ) }
$$

\section{$\mathrm{Bit} / \mathrm{s} / \mathrm{Hz}$}

Thus the theoretical capacity willbe:

$$
\begin{aligned}
& \text { C }=E\left(\notin \neq \log _{2}\left(1+\operatorname{SNR} \cdot E\left(|h|^{2}\right)\right)\right. \\
& \text { or } E\left(|h|^{2}\right)=1 \\
& \text { Thus: } \quad \mathbf{C}=\log _{2}(1+\operatorname{SNR}) \\
& \text { [6] }
\end{aligned}
$$$$
\text { The capacity increases as logarithmic function of }(1+\mathrm{SNR}) \text {. }
$$

\section{B. Capacity of SIMO Channel}

A channel SIMO (Single Input, Multiple Output) is multirecieving wire framework with $\mathrm{N}$ transmitting reception apparatus and $\mathrm{M}$ accepting radio wires. With the intricate addition between the transmit recieving wire and their getting radio wire, its ability will be $: C=\log _{2}(1+$ SNR.

$\left.\sum_{\substack{\mathrm{i}=1 \\ \mathrm{i}=1}}^{\mathrm{h}}\left|\mathrm{h}_{\mathrm{i}}\right|^{2}\right)$

$\left|h_{\mathrm{i}}\right|^{2}=M^{2}$, its Shannon capacity is given by:

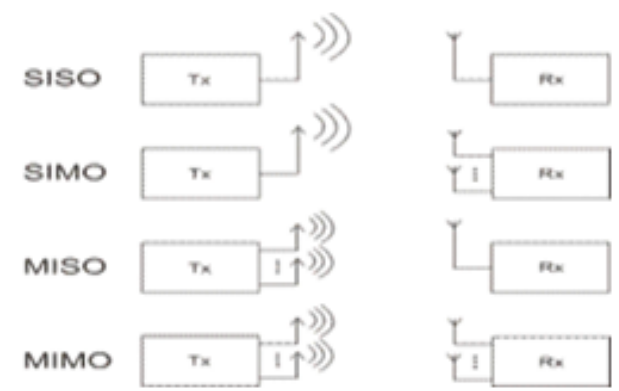

Fig.2. SISO, SIMO, MISO, MIMO Channels

In this division we will depict declaration remote correspondence frameworks Fig.2 and we will

$$
\mathrm{C}_{\text {theo }}=\mathrm{E}(\mathrm{C})=\log _{2}\left(1+\mathrm{SNR} \cdot \mathrm{M}^{2}\right)(12)
$$

We find that its capability add to with $\log$ of $(1+$ SNR M $\left.{ }^{2}\right)$, faster than in SISO case.

\section{Capacity of MIMO Channel}

With MIMO framework numerous recieving wires are utilized both at source recipient, the expansion of various reception apparatuses at the transmitter joined with cutting edge signal

handling calculations at source collector gives presentation as far as limit and diversity[10]. A MIMO channel is remote connection between $\mathrm{N}$ transmit and $\mathrm{M}$ get radio wires. MIMO involves NM components that establishes MIMO channel coefficients. 
The various transmit and get radio wires could have place with solitary client modem or it could be appropriated among various clients [10]. The channel grid $\mathrm{H}(\mathrm{N} \times \mathrm{M}$.) for MIMO channel with $\mathrm{N}$ transmit recieving wires $\mathrm{M}$ get reception apparatuses,

$$
\mathrm{H}=\left[\begin{array}{ccc}
\mathbf{h}_{11} & \cdots & \mathbf{h}_{1 \mathrm{~N}} \\
\vdots & \ddots & \vdots \\
\mathrm{H}_{\mathrm{M} 1} & \cdots \mathrm{h}_{\mathrm{MN}} &
\end{array}\right]
$$

Hijis the intricate channel gain between jth transmit reception apparatus ith receiving radio wire:

$$
\mathrm{C}=\log _{2}\left(\operatorname{det}\left[\mathrm{I}_{\mathbf{M}}+\operatorname{SNR} \frac{\mathrm{HH}^{\mathrm{H}}}{\mathrm{N}}\right]\right)
$$

In this condition, "det "signifies determinant, $I \mathrm{M}$ implies $\mathrm{N} \times \mathrm{M}$ character grid and $H \mathrm{H}$ means Matrix transpose conjugate where quantities receiving wires $\mathrm{N}$ and $\mathrm{M}$ are significant, Expected worth capacity limit with respect to Rayleigh channel develops relatively to $\mathrm{M}$.

$\mathrm{E}[\mathrm{C}] \approx \mathrm{M} \cdot \log _{2}(1+\mathrm{SNR}) \mathrm{Bit} / \mathrm{s} / \mathrm{Hz}$

Taking everything into account, Capacity increments at an lot quicker rate in MIMO frameworks than in SISO and SIMO because huge number radio wires case.

\section{COMPARISON OF CAPACITY FOR SISO, MISO, SIMO and MIMO}

Fig.3 demonstrates limit SISO and MIMO frameworks as far as SNR for various estimations $\mathrm{N}$ and $\mathrm{M}$. [15]. In the SISO structure $(\mathrm{N}=1$ and $\mathrm{M}=1)$ channel limit ranges from 1 to 17 bps/Hz. It stays low and increments bit by bit with SNR, which represents restrictions of SISO transmissions. In spite present procedures to benefit as much as possible from SISO channel limit stays restricted multi-radio wire framework, even underabused, gives better execution. In MIMO structures ( $\mathrm{N}=4$ and $\mathrm{M}=4$ ) limit stretches out from 3 to $48 \mathrm{bps} / \mathrm{Hz}$ (multiple times bigger than SISO. There is fast development with SNR, which exhibits presentation MIMO correspondence.

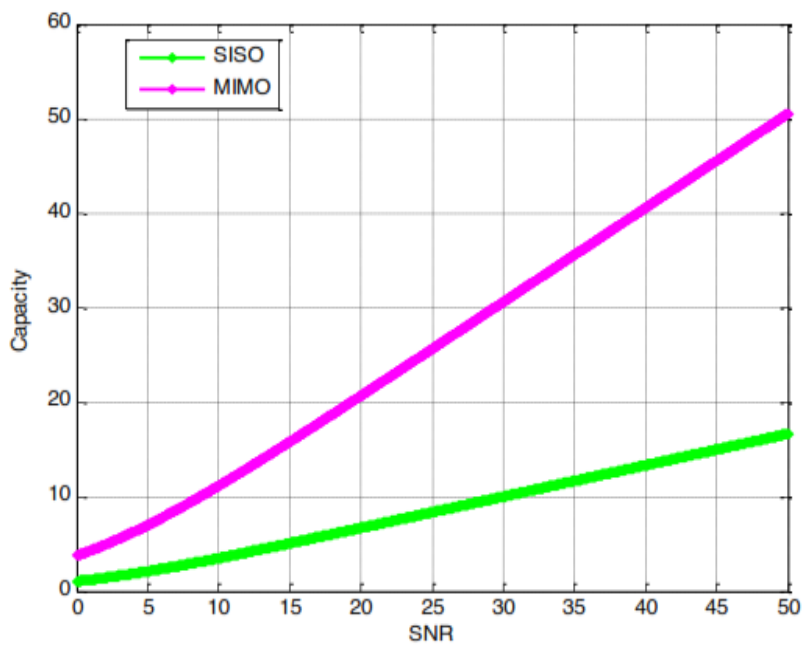

Fig. 3. Comparison in Capacity between SISO and MIMO.

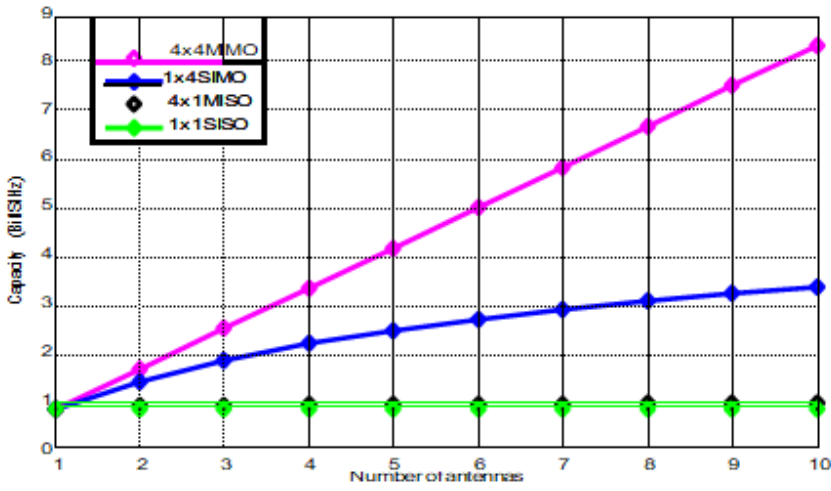

Fig. 4.Capacity vs number of antennas forMIMO, SIMO, MISO and SISO systems.

To show the expansion of limit as indicated by the quantity of receiving wires for all frameworks we give the accompanying (Fig.4) [8]:

When we take a gander at Figures 3 and 4, we see that MIMO limit develops directly as for the quantity of radio wires nearly $M$ times bigger than SISO limit ( $M$ number receiving wires). For MISO, presentation addition is immaterial when quantity transmit recieving wires more. SIMO structure is more viable and effective than SISO and MISO framework whose limit ranges from 1 to 3.4 bps/Hz. what's more, different stays associated with worth $1 \mathrm{bps} / \mathrm{Hz}$; it stays low with expanding number reception apparatuses, which clarifies restrictions SISO MISO transmissions. varieties limit SIMO and MIMO frameworks can be thought about dependent on quantity reception apparatuses for SNR of $15 \mathrm{~dB}$ (for example).Improved limit is progressively articulated for high SNR. For SIMO framework, limit pursues logarithmic pattern when we increase number of recieving wires at gathering while MIMO framework increments straightly when we increment quantity transmit and receive radio wires.

The benefit MIMO frameworks is fundamentally because abuse multipath. To start with, beneficiary is permitted recognize distinctive transmitting recieving wires, thusly to all while various images. At that point, every way is a copy of the transmitted sign, and hence conveys valuable data. It can likewise be said that every way is indistinguishable from the immediate sign transmitted by a virtual radio wire, that builds the quantity of transmit recieving wires for all intents and purposes. The cost to pay for this expansion in limit is first material, with the multiplication of reception apparatuses and their related hardware, yet additionally programming with considerably more perplexing collectors and requesting more power estimation and thusly expanded utilization. At last, corruption connection coefficient among the reception apparatuses can bring about decreased capacity. By expanding the quantity of radio wires for some MIMO frameworks limit increments with, and we have the accompanying (Fig.5): 


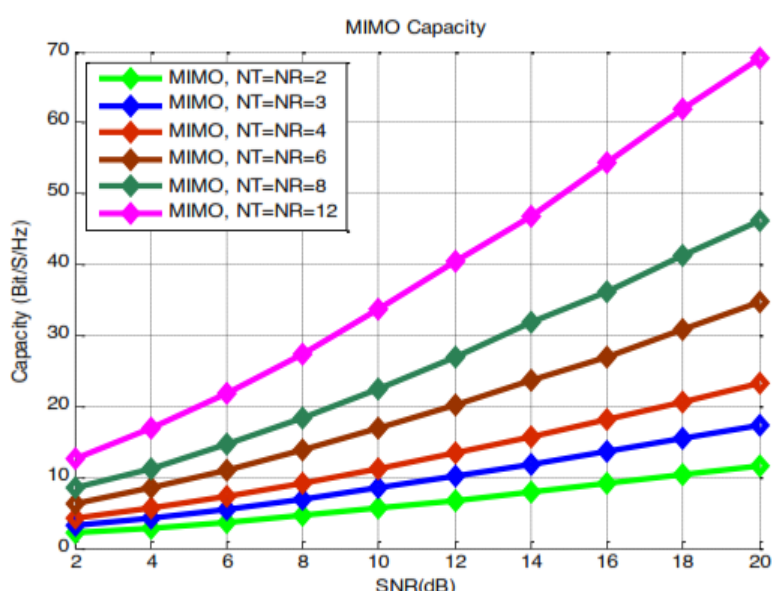

Fig.5. capacityvs Number of MIMO antennas

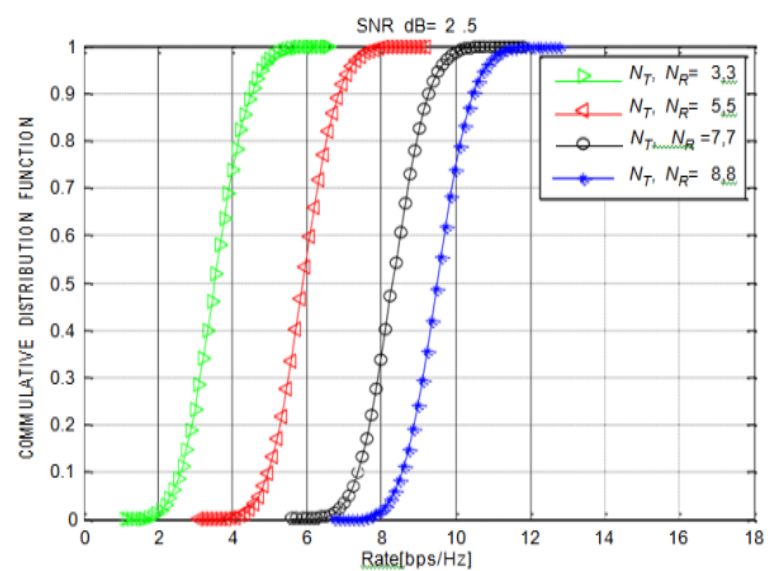

Fig.6. Shanon capacity vs SNR

Limit increments straightly with for $\min (\mathrm{Nt}, \mathrm{Nr})$ for given fixed transmitter power and data transfer capacity in MIMO framework.

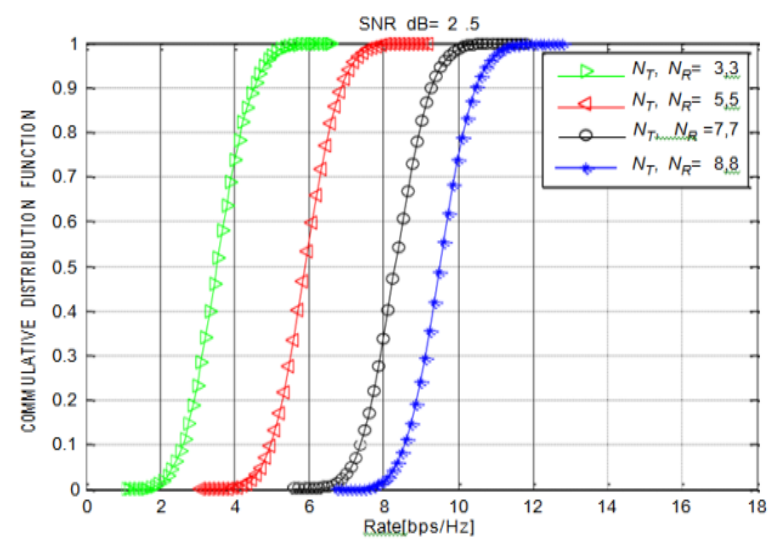

Fig. 7. Distribution of MIMO channel capacity

Total dispersion capacity of MIMO increments in result that we increment quantity transmitter and beneficiary.

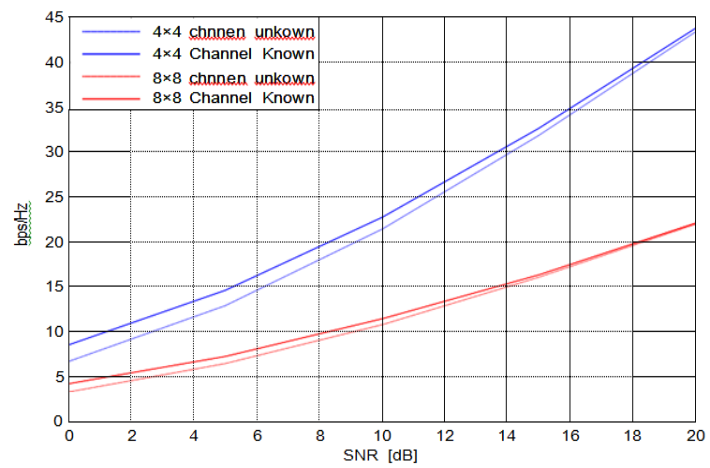

Fig. 8. Channel unknown and channel known

When we compare these three pairs of antennas $\left(N_{\mathrm{T}}=M_{\mathrm{R}}=2\right),\left(N_{\mathrm{T}}=M_{\mathrm{R}}=4\right)$ and $\left(N_{\mathrm{T}}=M_{\mathrm{R}}=3\right),\left(N_{\mathrm{T}}=M_{\mathrm{R}}=6\right)$ and $\left(N_{\mathrm{T}}=M_{\mathrm{R}}=4\right),\left(N_{\mathrm{T}}=M_{\mathrm{R}}=8\right)$ it is found to MIMO faculty is twice ; MIMO capacity then boost more quickly with SNR ,to close with over more than $50 \%$ at 20dBSNR. It has been shown dependency of MIMO channel capacity on channel knowledge .After that when we look pair antennas $\left(N_{\mathrm{T}}=M_{\mathrm{R}}=6\right),\left(N_{\mathrm{T}}=M_{\mathrm{R}}=12\right) \mathrm{MIMO}$ capacity then increases more rapidly with SNR, to finish with gainofmorethan 70\%at20dBSNR.

\section{Conclusion}

In this paper, a reasonable picture of the impact of using diverse reaction apparatus on the limit of remote correspondence frameworks is given by utilizing Matlabcode. Results created demonstrate the connection between number radio wire and limit; our outcomes demonstrate that expanding the quantity of transmitting and accepting reception apparatus for remote MIMO Channel undoubtedly improves the channel limit and execution that can be gotten.

Future remote correspondence frameworks will play out an achievement in framework execution, by taking utilization of receiving wire clusters at the two sides of correspondence connect.

\section{REFERENCE}

1. Lekun lin "choosing between open- and closed-loop mimo in bts systems" embedded computing design — may 15, 2009

2. David gesbert, member, ieee, mansoorshafi, fellow, ieee, dashanshiu, member, ieee, peter j. Smith, member, ieee, and aymannaguib, senior member, ieee " from theory to practice: an overview of mimo space-time coded wireless systems" ieee journa on selected areas in communications, vol. 21, no. 3, april 2003.

3. M. A. Jensen and j. W. Wallace, "a review of antennas and propagation for mimo wireless systems, "ieee trans. Antennas propagat., vol. 52, no. 11, pp. 2810-2824, nov. 2004

4. M. Bialkowski, "research into multiple element antennas to enhance performance of wireless communication systems," in proc. Xvi intrnl.conf.on microwaves, radar, wireless communications, vol. 3 , may 2006, pp. 1071-1082.

5. Ian poole, radio-electronics: resources and analysis for electronics engineers: what is mimo? Multiple input multiple out put tutorial.

6. E. Telater, "capacity of multi-antenna gaussian channel", at\&t bell labs, tech. Memo., june 1995.

7. E. Telatar, "capacity of multi-antenna gaussian channels", invited paper, european transactions on telecommunications, vol. Ett-10, no. 6, pp. 585-595, 1999.

8. Tim brown, elisabeth de carvalho, persefonikyritsi "practical guide to the mimo radio channel with matlab examples" this edition first published 2012

9. C. E. Shannon, "a mathematical theory of communication", bell syst. Techn. J., vol. 27, pp. 379-423 623-656, july, october, 1948 
10. Nirmalendubikassinha, r. Bera, m. Mitra“ capacity and v- blast techniques for mimo wireless" journal of theoretical and applied information technology 2005 - 2010 jatit.11.g. J. Foschini and m. J. Gans, "on limits of wireless communications in a fading

11. Environment when using multiple antennas,"

12. 12.' i. E. Telatar, "capacity of multi-antenna gaussian channels," eur. Trans. Telecomm., vol. 10, pp. 2172-2178, 2000.

13. Vasanthanraghavan and akbar $\mathrm{m}$. Sayeed "multi-antenna capacity of sparse multipath channels" information theory workshop 2004 (itw 2004), san antonio, tx and the ieee international symposium on information theory 2006 (isit 2006), seattle, wa.

14. Rafik ahmad, deveshpratap singh, mitali singh "ergodic capacity of mimo channel in multipath fading environment" i.j. Information engineering and electronic business, 2013, 3, 41-48 published online september 2013 in mecs.

15. Akhilesh kumar, anil chaudhary "channel capacity enhancement of wireless communication using mimo technology" international journal of scientific \& technology research volume 1 , issue 2 , march 2012. 